\title{
改进的灰色模型在光催化数据预测中的应用
}

\author{
王路平 ${ }^{1}$, 卢占会 ${ }^{1}$, 魏 金金 ${ }^{2}$, 方 明 ${ }^{3}$, 王祥科 ${ }^{3}$
}

(华北电力大学 1. 数理学院; 2. 控制与计算机工程学院; 3. 环境科学与工程学院, 资源环境系统优化教育部重点 实验室, 北京 102206)

摘 要: 光催化去除水中污染物的研究通常得到的是小样本的离散数据, 利用拟一级动力学模型对实验结果进行模 拟和分析, 有时拟合效果较差, 且无法用于数据预测。本研究在离散灰色预测模型 $(\operatorname{DGM}(1,1))$ 的基础上, 考虑数据 的非线性特征并结合等维信息替代思想建立了非线性动态离散灰色模型 $(\operatorname{EDGM}(1,1, \alpha))$, 利用该模型对三元复合 材料 $\mathrm{Bi} / \mathrm{BiOCl} / \mathrm{Au}$ 光催化去除四环素实验所得数据进行了预测, 其平均相对误差和拟合度数据显示: 相比于 $\operatorname{DGM}(1,1)$ 等 3 种模型, $\operatorname{EDGM}(1,1, \alpha)$ 模型对光催化实验数据具有良好的预测水平，与实验结果吻合。该预测方法 可以用于指导下一步实验, 有望减少实验次数, 降低成本和能耗。

关 键 词: 光催化; 预测; 灰色模型; 等维信息替代

中图分类号: TQ174 文献标志码: A

\section{Application of Improved Grey Model in Photocatalytic Data Prediction}

\author{
WANG Luping ${ }^{1}$, LU Zhanhui ${ }^{1}$, WEI Xin ${ }^{2}$, FANG Ming ${ }^{3}$, WANG Xiangke ${ }^{3}$
}

(1. School of Mathematics and Physics, North China Electric Power University, Beijing 102206, China; 2. College of Control and Computer Engineering, North China Electric Power University, Beijing 102206, China; 3. MOE Key Laboratory of Resources and Environmental System Optimization, College of Environmental Science and Engineering, North China Electric Power University, Beijing 102206, China)

\begin{abstract}
Discrete and small samples data are usually obtained from the studies on photocatalytic removal of pollutants in water. The first-order kinetic model used to simulate and analyze the experimental data sometimes has poor fitting effect and cannot be used for data prediction. In this study, based on the discrete grey model $(\mathrm{DGM}(1,1))$, the nonlinear dynamic discrete gray model $(\operatorname{EDGM}(1,1, \alpha))$ are established by considering the nonlinear characteristics of the data and equal dimensional information substitution method. The model is used to predict the experimental data of photocatalytic degradation of tetracycline by $\mathrm{Bi} / \mathrm{BiOCl} / \mathrm{Au}$. As compared with $\mathrm{DGM}(1,1)$ and other three models, the $\operatorname{EDGM}(1,1, \alpha)$ model has a better prediction level for the experimental data of photocatalysis. The results are in good agreement with the experimental results. This model can be used to guide the next step of experiments, which is expected to reduce the number of experiments and realize the rapid development of experimental research with low cost and energy consumption.
\end{abstract}

Key words: photocatalysis; predict; grey model; equivalent substitution method

收稿日期：2020-11-19; 收到修改稿日期：2021-02-04; 网络出版日期：2021-03-01

基金项目: 科技部重大研发计划(2018YFC1900105, 2017YFA0207002); 中央高校基本科研业务费专项资金(2019MS040) National Key Research and Development Program of China (2018YFC1900105, 2017YFA0207002); Fundamental Research Funds for Central Universities (2019MS040)

作者简介: 王路平(1996-), 女, 硕士研究生. E-mail: lupingwang@ncepu.edu.cn WANG Luping (1996-), female, Master candidate. E-mail: lupingwang@ncepu.edu.cn

通信作者：卢占会，教授.E-mail: luzhanhui@ncepu.edu.cn; 王祥科，教授.E-mail: xkwang@ncepu.edu.cn LU Zhanhui, professor. E-mail: luzhanhui@ncepu.edu.cn; WANG Xiangke, professor. E-mail: xkwang@ncepu.edu.cn 
光催化技术以其无二次污染、成本低、效率高 等特点被用于去除水中的有机污染物、重金属污染 物、微生物等, 引起了广泛关注 ${ }^{[1-5]}$ 。与实验技术的 迅速发展相比, 光催化的数据拟合与预测的研究非 常少。常用的光催化数据处理模型一般为拟一级动 力学模型, 该模型在拟合数据和分析速率时效果不 佳 $^{[6-7]}$ 。另外, 还未见基于有限数据预测持续反应过 程的研究报道。实验数据的拟合、分析及预测对深 入理解光催化降解实验过程有重要意义。

传统的统计预测模型, 大多是基于半经验数学 模型设计 ${ }^{[8-9]}$, 必须在基础理论模型上加入实验 数据进行修正。现代预测方法只是因果关系的表达 或对未来趋势的说明 ${ }^{[10]}$ 。准确开展以上方法准需要 处理大量不同类型数据, 得到所需实验数据的工作 量巨大 ${ }^{[11]}$ 。灰色模型 $(G M(1,1)$ 模型)在利用少量实 验数据进行预测方面显示了它的实用性, 并已应用 于多个领域 ${ }^{[12-17]}$ 。现有多种方法用于灰色模型的改 进与优化, 包括残差校正法、背景值构造法、等维 信息替代法、中心法、时间响应法等 ${ }^{[18-19]}$ 。等维信 息替代模型又称代谢模型, 即用最新数据替换初 始数据, 每次预测都会修正参数, 从而提高整体预 测精度。

由于光催化采集的实验数据是离散的, 反应速 率不固定, 因此在离散灰色预测模型 $(\operatorname{DGM}(1,1)$ 模 型)基础上建立非线性离散灰色预测模型(DGM(1, 1, $\alpha)$ 模型)更符合光催化降解实验数据特点。在使用 $\operatorname{DGM}(1,1, \alpha)$ 模型时可以根据不同实验数据改变 $\alpha$, 从而提高拟合度。本研究还结合光催化反应过程的动 态性特点, 提高近距离数据在预测过程中的权重, 建立等维信息替代非线性离散灰色模型 $\operatorname{EDGM}(1$, $1, \alpha)$ ), 该模型对实验数据拟合效果佳。

\section{DGM(1, 1)模型和分析}

光催化是在光照射下, 光催化材料吸收光以后 引起化学反应或者改变初始反应的速率, 使得化学 成分改变的反应。反应过程受多方面因素的影响并 且获得的数据样本较少。而灰色预测模型对解决这 类有先验知识或经验的超小样本的建模问题具有较 好的实用意义。2005 年, 谢乃明提出了离散灰色模型 $(\operatorname{DGM}(1,1))$, 将参数估计和预测模型统一为离散形 式 ${ }^{[20]}$ 。DGM(1, 1)模型可以避免 $\mathrm{GM}(1,1)$ 模型将离 散序列转化为连续序列过程中的误差, 更适用于预测 光催化数据 ${ }^{[21]}$ 。 $\operatorname{DGM}(1,1)$ 建模思想如下 ${ }^{[22-23]}$ :
假设 $x^{(0)}(t)=\left\{x^{(0)}(1), x^{(0)}(2), \cdots, x^{(0)}(N)\right\}$ 为原始 时间序列, 累加生成 $x^{(1)}(t)$ :

$$
x^{(1)}(t)=\sum_{i=1}^{t} x^{(0)}(i)
$$

得到: $x^{(1)}(t)=\left\{x^{(1)}(1), x^{(1)}(2), \cdots, x^{(1)}(N)\right\}$, 则 $\operatorname{DGM}(1,1)$ 模型为 ${ }^{[24]}$ :

$$
x^{(1)}(t+1)=\beta_{1} x^{(1)}(t)+\beta_{2}
$$

$\beta_{1}, \beta_{2}$ 是被估计的参数, 估计值是:

$$
\left[\begin{array}{l}
\widehat{\beta_{1}} \\
\widehat{\beta_{2}}
\end{array}\right]=\left(X^{T} X\right)^{-1} X^{T} Y
$$

其中

$$
X=\left[\begin{array}{cc}
x^{(1)}(1) & 1 \\
x^{(1)}(2) & 1 \\
\cdots & \ldots \\
x^{(1)}(N-1) & 1
\end{array}\right], Y=\left[\begin{array}{c}
x^{(1)}(2) \\
x^{(1)}(3) \\
\cdots \\
x^{(1)}(N)
\end{array}\right]
$$

若初始条件为 $x^{(1)}(1)=x^{(0)}(1)$, 得到(2)的递归 函数为:

$$
\hat{x}^{(1)}(t+1)=\beta_{1}^{t}\left(x^{(0)}(1)-\frac{\beta_{2}}{1-\beta_{1}}\right)+\frac{\beta_{2}}{1-\beta_{1}}
$$

由式(2)和式(5)得到 $x^{(0)}(t)$ 的预测值:

$$
\hat{x}^{(0)}(t+1)=\left(\beta_{1}^{t}-\beta_{1}^{t-1}\right)\left(x^{(0)}(1)-\frac{\beta_{2}}{1-\beta_{1}}\right)
$$

最后, 讨论 $\operatorname{DGM}(1,1)$ 模型增长率 $\lambda$

$$
\begin{gathered}
\hat{x}^{(0)}(k+1)=\hat{x}^{(1)}(k+1)-\hat{x}^{(1)}(k)= \\
\left(\beta_{1}^{t}-\beta_{1}^{t-1}\right)\left(x^{(0)}(1)-\frac{\beta_{2}}{1-\beta_{1}}\right) \\
\lambda=\frac{\hat{x}^{(0)}(k+1)-\hat{x}^{(0)}(k)}{\hat{x}^{(0)}(k)}=\beta_{1}-1
\end{gathered}
$$

可见增长率 $\lambda$ 是一个常数。这与光催化实验数 据的非线性特征不相符, 因此必须对该模型进行 改进。

\section{$2 \operatorname{DGM}(1,1)$ 模型的改进}

\subsection{DGM $(1,1, \alpha)$ 模型的建立}

反应过程中所得实验数据呈现非线性关系, 将前述 $\operatorname{DGM}(1,1)$ 的 (2) 式改进为 $x^{(1)}(t+1)=$ $\beta_{1}\left(x^{(1)}(t)\right)^{\alpha}+\beta_{2}$, 建立 $\operatorname{DGM}(1,1, \alpha)$ 模型。通过改变 $\alpha$, 提高预测效果, $\operatorname{DGM}(1,1, \alpha)$ 建模思想如下: 
假设 $x^{(0)}(t)=\left\{x^{(0)}(1), x^{(0)}(2), \cdots, x^{(0)}(N)\right\}$ 为原始 时间序列，累加生成 $x^{(1)}(t)$ :

$$
x^{(1)}(t)=\sum_{i=1}^{t} x^{(0)}(i)
$$

得到： $x^{(1)}(t)=\left\{x^{(1)}(1), x^{(1)}(2), \cdots, x^{(1)}(N)\right\}$, 建立 DGM $(1,1, \alpha)$ 模型:

$$
\left\{\begin{array}{l}
x^{(1)}(t+1)=\beta_{1}\left(x^{(1)}(t)\right)^{\alpha}+\beta_{2} \\
x^{(1)}(1)=x^{(0)}(1)
\end{array}\right.
$$

$\beta_{1}, \beta_{2}$ 是被估计的参数, 估计值是:

$$
\left[\begin{array}{l}
\widehat{\beta}_{1} \\
\widehat{\beta_{2}}
\end{array}\right]=\left(X^{T} X\right)^{-1} X^{T} Y
$$

其中

$$
X=\left[\begin{array}{cc}
\left(x^{(1)}(1)\right)^{\alpha} & 1 \\
\left(x^{(1)}(2)\right)^{\alpha} & 1 \\
\ldots & \ldots \\
\left(x^{(1)}(N-1)\right)^{\alpha} & 1
\end{array}\right], Y=\left[\begin{array}{c}
x^{(1)}(2) \\
x^{(1)}(3) \\
\ldots \\
x^{(1)}(N)
\end{array}\right]
$$

$\operatorname{DGM}(1,1, \alpha)$ 模型和 $\operatorname{DGM}(1,1)$ 模型的区别在 于要求 $\alpha$ 的最优值, 只有得到 $\alpha$ 的最优值, 才可以 使得预测达到最大精度。在寻找 $\alpha$ 最优值过程中, 选 取平均相对误差为预测指标, 以确定的步长不断改 变 $\alpha$,从而寻找最优 $\alpha$ 。

$\operatorname{DGM}(1,1, \alpha)$ 模型步骤如下:

(1)建立非线性 $\operatorname{DGM}(1,1, \alpha)$ 模型

$$
\left\{\begin{array}{l}
x^{(1)}(t+1)=\beta_{1}\left(x^{(1)}(t)\right)^{\alpha}+\beta_{2} \\
x^{(1)}(1)=x^{(0)}(1)
\end{array}\right.
$$

(2)求最优 $\alpha$ 。

设置目标函数为预测数据和原始数据的最小平 均相对误差, 给定 $\alpha$ 初始值, 并设置搜索步长, 根 据预测数据和原始数据的平均相对误差动态调整 $\alpha$, 直至得到最优 $\alpha$ 。

(3)将 $\alpha$ 代入 $\operatorname{DGM}(1,1, \alpha)$ 模型求 $\beta_{1}, \beta_{2}$ 。

(4)求得 $\hat{x}^{(1)}(k)$ 和 $\hat{x}^{(0)}(k)$ 。

(5)进行预测。

$\alpha$ 可以随不同数据的变化而变化, $\alpha>1$, 反应速 度越来越快; $\alpha<1$, 反应速度越来越慢; $\alpha=1$, 即为 $\operatorname{DGM}(1,1)$ 模型。因此 $\operatorname{DGM}(1,1, \alpha)$ 模型应用范围更 广一些, 可以适应更复杂的数据预测。

\section{$2.2 \operatorname{EDGM}(1,1)$ 模型的建立}

随着时间的推移, 影响光催化反应的因素不断 变化, 系统的状态也在不断变化。如果仍使用初始 数据进行预测, 不考虑动态变化的特性, 预测的可 靠性也会下降。在一个时间连续且不断预测新数据 的系统中, 将预测到的数据点应用于下一个数据点 的预测中, 每得到一个新的预测值 $x^{(0)}(N+1)$, 就 将该预测值加入到 $x^{(0)}(t)$ 数据序列中, 并将 $x^{(0)}(1)$ 数据点去除, 生成新的数据序列用于下一个数据点 的预测，该思想称为等维信息替代思想。将等维信 息替代思想与 $\operatorname{DGM}(1,1)$ 模型相结合就得到了 $\operatorname{EDGM}(1,1)$ 模型, 该模型步骤如下:

(1)选择 $x^{(0)}(t)=\left\{x^{(0)}(1), x^{(0)}(2), \cdots, x^{(0)}(N)\right\}$ 为原 始时间序列。

(2) 建立 DGM $(1,1)$ 模型, 并预测 $x^{(0)}(N+1)$ 的值。

(3)更新数据: 删除 $x^{(0)}(1)$, 新增数据 $x^{(0)}(N+1)$, 得到 $x^{(0)}(t)=\left\{x^{(0)}(2), x^{(0)}(3), \cdots, x^{(0)}(N+1)\right\}$ 。

(4)利用新的数据序列建立 $\operatorname{DGM}(1,1)$ 模型, 并 对 $x^{(0)}(N+2)$ 值进行预测。

(5)重复上述步骤, 直到完成预测。

\section{$2.3 \operatorname{EDGM}(1,1, \alpha)$ 模型的建立}

将 $\operatorname{EDGM}(1,1)$ 模型和 $\operatorname{DGM}(1,1, \alpha)$ 模型结合, 得到 $\operatorname{EDGM}(1,1, \alpha)$ 模型，建模过程如下：

(1)选择 $x^{(0)}(t)=\left\{x^{(0)}(1), x^{(0)}(2), \cdots, x^{(0)}(N)\right\}$ 为原 始时间序列。

(2)建立 $\operatorname{DGM}(1,1, \alpha)$ 模型, 并预测 $x^{(0)}(N+1)$ 的值。

(3)更新数据: 删除 $x^{(0)}(1)$, 新增数据 $x^{(0)}(N+1)$ 。

(4)利用新的数据序列建立新的 $\operatorname{DGM}(1,1, \alpha)$ 模 型, 求出新的 $\alpha$, 预测 $x^{(0)}(N+2)$ 。

(5)重复上述步骤, 直到完成预测。

$\operatorname{EDGM}(1,1, \alpha)$ 模型步骤如图 1 所示, 不断加入 新的数据, 计算新的最优 $\alpha$, 使得模型具有动态更 新的特点。 $\operatorname{EDGM}(1,1, \alpha)$ 与 $\operatorname{DGM}(1,1)$ 相比最大的 优势在于将静态模型转化为动态模型, 减少静态模 型带来的误差。 $\operatorname{EDGM}(1,1, \alpha)$ 模型是在 $\operatorname{DGM}(1,1)$ 模型基础上建立起来的, 因此要求原始数据累加后 具有递增或递减趋势。

\section{4 模型检验方法}

为检验模型的预测效果, 选择平均相对误差 (MRE): 


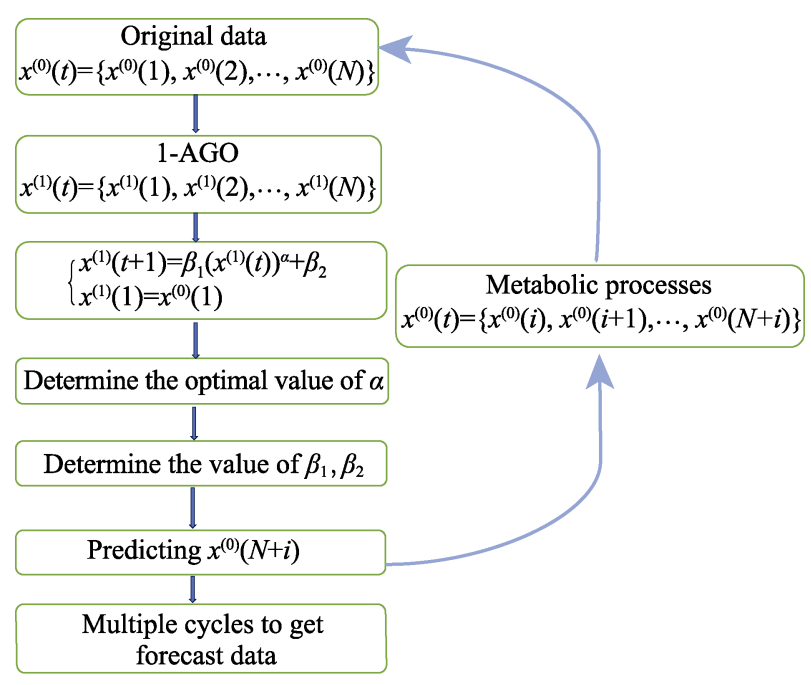

图 $1 \operatorname{EDGM}(1,1, \alpha)$ 的建模步骤

Fig. 1 Modelling steps of $\operatorname{EDGM}(1,1, \alpha)$

$$
\operatorname{MRE}=\frac{1}{n} \sum_{k=1}^{N}\left|\frac{\hat{x}^{(0)}(k)-x^{(0)}(k)}{x^{(0)}(k)}\right|
$$

和拟合度(FD):

$$
\mathrm{FD}=1-\frac{\sqrt{\sum_{k=1}^{N}\left[\hat{x}^{(0)}(k)-x^{(0)}(k)\right]^{2}}}{\sqrt{\sum_{k=1}^{N} \hat{x}^{(0)}(k)^{2}}+\sqrt{\sum_{k=1}^{N} x^{(0)}(k)^{2}}}
$$

来评估模型的预测精度。其中, $\hat{x}^{(0)}(k)$ 为预测值, $x^{(0)}(k)$ 为原始值。MRE 越小, FD 越大, 模型精度越高。

\section{3 实例分析}

\section{1 实验数据的获取}

\subsubsection{Bi/BiOCl/Au 的制备}

所用药品均为分析纯。按照文献[25]的方法制 备 $\mathrm{Bi} / \mathrm{BiOCl}$ : 取 $1 \mathrm{~g} \mathrm{Bi/BiOCl}$ 加入 $20 \mathrm{~mL}$ 超纯水中, 超声分散后加入 $0.01 \mathrm{~g}$ 氯金酸, 在搅拌状态下, 滴 加硼氢化钠至完全生成沉淀, 室温搅拌 $2 \mathrm{~h}$ 后, 用乙 醇洗净烘干得到 $\mathrm{Bi} / \mathrm{BiOCl} / \mathrm{Au}$ 。

\subsubsection{Bi/BiOCl/Au 表征}

利用紫外 - 可见分光光光度计 UV-2700 (Shimadzu, UV-2700)测量样品的紫外-可见吸收谱 图。用 SmartLab SE (Rigaku Corporation, $\mathrm{Cu} \mathrm{K} \alpha$ ) 型 X 射线衍射仪分析样品的晶相。

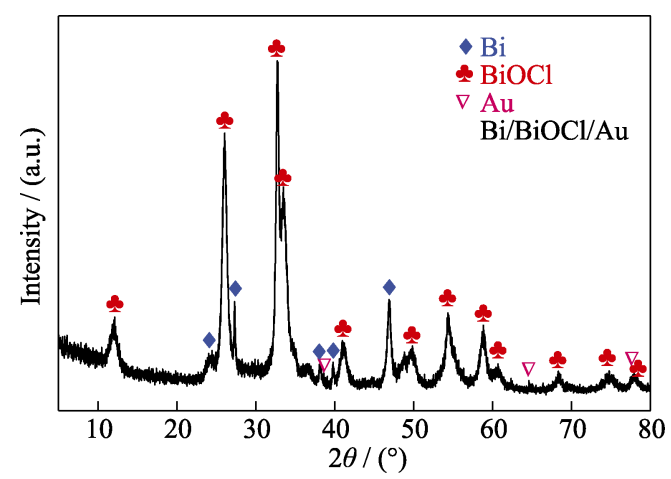

图 $2 \mathrm{Bi} / \mathrm{BiOCl} / \mathrm{Au}$ 的 XRD 谱图

Fig. 2 XRD pattern of $\mathrm{Bi} / \mathrm{BiOCl} / \mathrm{Au}$

图 2 展示了 $\mathrm{Bi} / \mathrm{BiOCl} / \mathrm{Au}$ 的 XRD 图谱, 由图 2 可知, XRD 图谱中出现了 Bi(JCPDs 44-1246)和 $\mathrm{BiOCl}$ 的特征峰(JCPDs 06-0249) ${ }^{[25]}$ 。Au 的特征峰位 于 $2 \theta=38.2^{\circ} 、 44.3^{\circ} 、 64.5^{\circ}$ 和 $78.2^{\circ}$, 与 $\mathrm{BiOCl}$ 或 $\mathrm{Bi}$ 的特征峰重合。无杂质峰出现, 表明实验制备了纯 $\mathrm{Bi} / \mathrm{BiOCl} / \mathrm{Au}$ 。

\subsection{3 光催化实验}

将 $50 \mathrm{mg} \mathrm{Bi} / \mathrm{BiOCl} / \mathrm{Au}$ 放入 $50 \mathrm{~mL}$ 的四环素 $(50 \mathrm{mg} / \mathrm{L})$ 溶液中, 在黑暗中搅拌 $120 \mathrm{~min}$ 保证吸附解吸平衡, 然后打开配有可见光滤光片 (400 nm) 的 $500 \mathrm{~W} \mathrm{Xe}$ 灯, 每 15 min 取样一次。采用紫外-可见 吸收光谱仪, 在 $357 \mathrm{~nm}$ 处测试吸光度, 根据吸光度 值计算四环素浓度, 得到的时间-四环素浓度实验 数据如表 1 所示。

\section{2 实例验证}

为了寻找预测时原始数据的最佳规模, 选取 $4 \sim 8$ 个数据作为原始数据, 利用 $\operatorname{EDGM}(1,1, \alpha)$ 模型 向后预测 2 个数据, 不同数据规模预测比较如表 2 所示。由表 2 可知, 当原始数据量为 4 时, MRE 为 0.005 , 预测效果最好。因此选择 4 个数据作为原始 数据进行预测。

对 $\mathrm{Bi} / \mathrm{BiOCl} / \mathrm{Au}$ 光催化四环素实验所得数据进 行预测, 取前 4 个数据作为原始数据, 向后预测 6 个。将预测值和实验值汇总于表 3, MRE 和 FD 数据 汇总于表 4 。

由表 4 可知 $\operatorname{DGM}(1,1)$ 模型 MRE 为 0.032 , 是 4 个预测模型中的最大值。 $\operatorname{EDGM}(1,1, \alpha)$ 预测精度最 高, FD 达到了 0.995, MRE 仅有 0.008 。 $\operatorname{DGM}(1,1)$ 模型、 $\operatorname{DGM}(1,1, \alpha)$ 模型、 $\operatorname{EDGM}(1,1)$ 模型的 $\mathrm{FD}$ 分 别为 $0.984 、 0.985 、 0.988$ 。

表 1 时间-四环素浓度实验数据

Table 1 Experimental data of tetracycline concentration

\begin{tabular}{ccccccccccc}
\hline Time $/ \mathrm{min}$ & 0 & 15 & 30 & 45 & 60 & 75 & 90 & 105 & 120 & 135 \\
\hline Con. $/\left(\mathrm{mg} \cdot \mathrm{L}^{-1}\right)$ & 38.66 & 34.52 & 31.39 & 29.10 & 27.00 & 25.15 & 23.16 & 21.24 & 19.40 & 18.29 \\
\hline
\end{tabular}


表 2 不同数据规模预测比较

Table 2 Different data size forecast comparison

\begin{tabular}{clrrrr}
\hline Date size & \multicolumn{2}{c}{$\begin{array}{c}\text { Experimental } \\
\text { data }\left(\mathrm{mg} \cdot \mathrm{L}^{-1}\right)\end{array}$} & \multicolumn{2}{c}{$\begin{array}{c}\text { Forecast data } \\
/\left(\mathrm{mg} \cdot \mathrm{L}^{-1}\right)\end{array}$} & MRE \\
\hline 4 & 27.00 & 25.15 & 26.93 & 24.89 & 0.005 \\
5 & 25.15 & 23.16 & 25.13 & 23.42 & 0.006 \\
6 & 23.16 & 21.24 & 23.50 & 21.98 & 0.025 \\
7 & 21.24 & 19.40 & 21.46 & 19.84 & 0.017 \\
8 & 19.40 & 18.29 & 19.56 & 17.97 & 0.025 \\
\hline
\end{tabular}

表 $3 \operatorname{DGM}(1,1) 、 \operatorname{DGM}(1,1, \alpha) 、 \operatorname{EDGM}(1,1)$ 和 $\operatorname{EDGM}(1,1, \alpha)$ 模型预测值

Table 3 Predicted values of $\operatorname{DGM}(1,1), \operatorname{DGM}(1,1, \alpha)$, $\operatorname{EDGM}(1,1)$, and $\operatorname{EDGM}(1,1, \alpha)$ models

\begin{tabular}{rccccc}
\hline $\begin{array}{c}\text { Time } \\
/ \mathrm{min}\end{array}$ & $\begin{array}{c}\text { Con. } \\
/\left(\mathrm{mg} \cdot \mathrm{L}^{-1}\right)\end{array}$ & $\begin{array}{c}\text { DGM } \\
(1,1)\end{array}$ & $\begin{array}{c}\text { DGM } \\
(1,1, \alpha)\end{array}$ & $\begin{array}{c}\text { EDGM } \\
(1,1)\end{array}$ & $\begin{array}{c}\text { EDGM } \\
(1,1, \alpha)\end{array}$ \\
\hline 60 & 27.00 & 26.60 & 26.93 & 26.60 & 26.93 \\
75 & 25.15 & 24.41 & 25.07 & 24.57 & 24.89 \\
90 & 23.16 & 22.40 & 23.41 & 22.52 & 22.98 \\
105 & 21.24 & 20.55 & 21.92 & 20.75 & 21.20 \\
120 & 19.40 & 18.86 & 20.56 & 19.05 & 19.56 \\
135 & 18.29 & 17.31 & 19.32 & 17.54 & 18.03 \\
\hline
\end{tabular}

$\operatorname{DGM}(1,1, \alpha)$ 的 $\mathrm{FD}$ 为 0.985 仅比 $\operatorname{DGM}(1,1)$ 的 $\mathrm{FD}(0.984)$ 提高 0.001 , 这是由于没有考虑光催化反 应速率是时刻变化的。图 3 表示 4 个模型对四环素 浓度的预测结果。由图 3(b)知, 光催化 $100 \mathrm{~min}$ 后, $\operatorname{DGM}(1,1, \alpha)$ 模型的预测误差逐渐变大。为了直观展 示误差随预测时间的变化, 将预测值减去实验数值
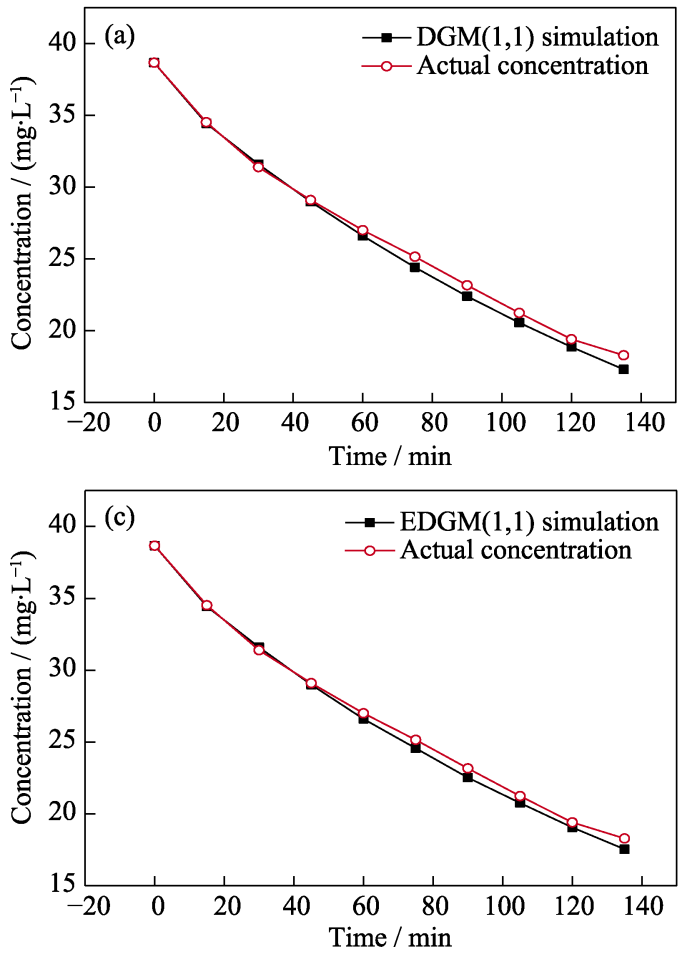

表 $4 \operatorname{DGM}(1,1) 、 \operatorname{DGM}(1,1, \alpha) 、 \operatorname{EDGM}(1,1)$ 和 $\operatorname{EDGM}(1,1, \alpha)$ 模型的 MRE 和 FD

Table 4 The MRE and FD of DGM(1, 1), DGM(1, 1, $\alpha)$, $\operatorname{EDGM}(1,1)$, and $\operatorname{EDGM}(1,1, \alpha)$ models

\begin{tabular}{ccc}
\hline Model & MRE & FD \\
\hline $\operatorname{DGM}(1,1)$ & 0.032 & 0.984 \\
$\operatorname{DGM}(1,1, \alpha)$ & 0.028 & 0.985 \\
$\operatorname{EDGM}(1,1)$ & 0.025 & 0.988 \\
$\operatorname{EDGM}(1,1, \alpha)$ & 0.008 & 0.995 \\
\hline
\end{tabular}

得到 4 个模型对四环素浓度的预测误差并汇总至 图 4。由图 4 可以看出, $\operatorname{DGM}(1,1, \alpha)$ 模型的预测误 差随预测时间的延长逐渐增大, 说明光催化反应速 率越来越慢, 预测时仅考虑到前 4 个数据的反应速 率, 得到的 $\alpha$ 会偏大。随着反应时间的延长, 实际反 应速率越来越慢, 模型精度也会越来越低。

$\operatorname{EDGM}(1,1)$ 模型考虑到光催化过程动态变化 的特点, 由图 4 知, $\operatorname{EDGM}(1,1)$ 模型的预测误差比 较平稳, 在-0.5 附近摆动。通过图 3(c) 可以看出, 虽 然 $\operatorname{EDGM}(1,1)$ 模型的误差波动较小, 但是整体误差 较大。由表 4 可以得到 $\operatorname{EDGM}(1,1)$ 模型预测的 MRE 为 0.025 , 仅比 $\operatorname{DGM}(1,1)$ 少了 0.007 。

$\operatorname{EDGM}(1,1, \alpha)$ 模型可以不断改变 $\alpha$ 的大小, $\alpha$ 分 别为 $1.019 、 0.996 、 0.997 、 0.998 、 0.999 、 0.999$ 。 由图 4 可知, $\operatorname{EDGM}(1,1, \alpha)$ 误差在 0 附近波动且较 小, 这是由于模型考虑到了光催化数据的动态特 征。由表 4 可知, $\operatorname{EDGM}(1,1, \alpha)$ 的 MRE 仅有 0.008 。
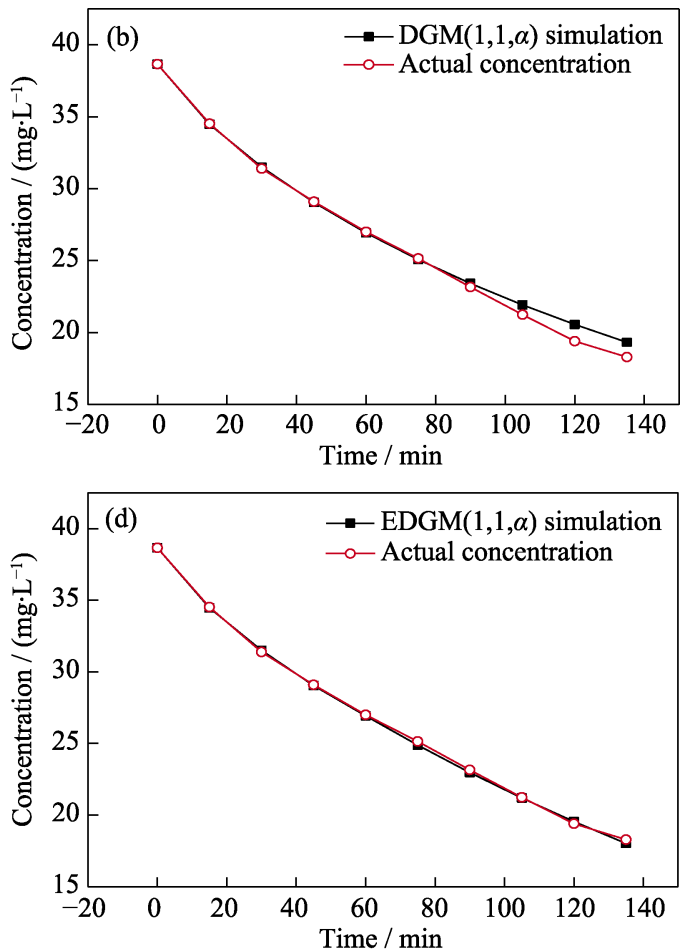

图 3 (a)DGM(1, 1), (b)DGM $(1,1, \alpha)$, (c)EDGM $(1,1),(d) \operatorname{EDGM}(1,1, \alpha)$ 模型对四环素浓度的预测结果

Fig. 3 Simulating results of tetracycline concentration from (a) $\operatorname{DGM}(1,1)$, (b) $\operatorname{DGM}(1,1, \alpha)$, (c) $\operatorname{EDGM}(1,1)$, and (d) $\operatorname{EDGM}(1,1, \alpha)$ models 


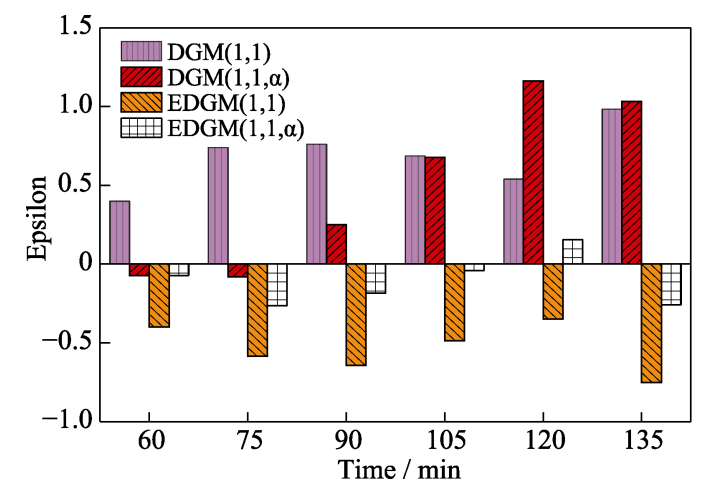

图 $4 \operatorname{DGM}(1,1), \operatorname{DGM}(1,1, \alpha), \operatorname{EDGM}(1,1), \operatorname{EDGM}(1,1, \alpha)$ 模型对四环素浓度的预测误差

Fig. 4 Simulated epsilon of tetracycline concentration from $\operatorname{DGM}(1,1), \operatorname{DGM}(1,1, \alpha), \operatorname{EDGM}(1,1)$, and $\operatorname{EDGM}(1,1, \alpha)$ models

$\operatorname{EDGM}(1,1, \alpha)$ 的 $\operatorname{FD}(0.995)$ 比 $\operatorname{DGM}(1,1)$ 的 $\operatorname{FD}(0.984)$ 提高了 0.011 。图 3(d)直观地展示了 $\operatorname{EDGM}(1,1, \alpha)$ 对光催化数据有最优的预测效果。

借助 $\operatorname{EDGM}(1,1, \alpha)$ 模型, 在实验室获取本组数 据时, 可将反应时间由 $135 \mathrm{~min}$ 缩短至 $60 \mathrm{~min}$, 大幅 缩短实验时间, 减少人力物力的损耗, 加快实验研 究的进程; 也可加大预测范围, 摆脱对实验室反应 仪器容量的限制, 寻找长时间反应的规律。

\section{3 结论}

本研究基于光催化过程的动态变化和光催化数 据的非线性特点, 在离散灰色预测模型基础上提出 了非线性离散灰色预测模型(DGM $(1,1, \alpha))$, 并将该 模型和等维信息替代思想结合, 得到了一种新的灰 色模型 $(\operatorname{EDGM}(1,1, \alpha))$ 用来预测光催化反应时间浓度数据序列, 并将该模型应用于 $\mathrm{Bi} / \mathrm{BiOCl} / \mathrm{Au}$ 光 催化降解四环素研究, 结果表明, 利用灰色模型对 $\mathrm{Bi} / \mathrm{BiOCl} / \mathrm{Au}$ 光催化降解四环素实验数据进行预测, 与 $\operatorname{DGM}(1,1) 、 \operatorname{DGM}(1,1, \alpha) 、 \operatorname{EDGM}(1,1)$ 模型相比, $\operatorname{EDGM}(1,1, \alpha)$ 的精确性明显提高, 各预测值的误差 较小且稳定, 说明对该模型的改进是有效的。

\section{参考文献:}

[1] ZHANG S, LI B, WANG X, et al. Recent developments of twodimensional graphene-based composites in visible-light photocatalysis for eliminating persistent organic pollutants from wastewater. Chemical Engineering Journal, 2020, 390: 124642.

[2] HAO M, QIU M, YANG H, et al. Recent advances on preparation and environmental applications of MOF-derived carbons in catalysis. Science of the Total Environment, 2021, 760: 143333.

[3] ZHANG Y, ZHU M, ZHANG S, et al. Highly efficient removal of $\mathrm{U}(\mathrm{VI})$ by the photoreduction of $\mathrm{SnO}_{2} / \mathrm{CdCO}_{3} / \mathrm{CdS}$ nanocomposite under visible light irradiation. Applied Catalysis B: Environmental, 2020, 279: 119390
[4] LIU X, PANG H, LIU X, et al. Orderly porous covalent organic frameworks-based materials: superior adsorbents for pollutants removal from aqueous solutions. The Innovation, 2021, 2: 100076.

[5] YAO L, YANG H, CHEN Z, et al. Bismuth oxychloride-based materials for the removal of organic pollutants in wastewater. Chemosphere, 2021, 273: 128576.

[6] WEI X, LU Z, WANG L, et al. Mechanism study of tetracycline high efficient photodegradation by $\mathrm{Bi}_{2} \mathrm{WO}_{6}$ nanosheets under visible light irradiation. Journal of Inorganic Materials, 2019, 35(3): 324-328.

[7] ZHU M, CAI Y, LIU S, et al. $\mathrm{K}_{2} \mathrm{Ti}_{6} \mathrm{O}_{13}$ hybridized graphene oxide: effective enhancement in photodegradation of Rh $\mathrm{B}$ and photoreduction of U(VI). Environmental Pollution, 2019, 248: 448-455.

[8] WHITE J A, VELASCO S. A simple semiempirical method for predicting the temperature-entropy saturation curve of pure fluids. Industrial and Engineering Chemistry Research, 2018, 58(2): 1308-1403.

[9] NAGUIB I A, ABDALLAH F F. Ultraviolet cutoff area and predictive ability of partial least squares regression method: a pharmaceutical case study. Spectrochimica Acta Part A: Molecular and Biomolecular Spectroscopy, 2020, 231: 118116.

[10] ZHAI M. A new method for short-term load forecasting based on fractal interpretation and wavelet analysis. International Journal of Electrical Power \& Energy Systems, 2015, 69: 241-245.

[11] CHENG M, SHI G, XIANG M. On the improvement of the parameter estimation of the grey model $\operatorname{GM}(1,1)$ and model application. Communications in Statistics-Simulation and Computation, 2020, 49(5): 1367-1384.

[12] DENG J. Control problems of grey systems. Systems \& Control Letters, 1982, 1(5): 288-294.

[13] ZHOU D, AL-DURRA A, ZHANG K, et al. A robust prognostic indicator for renewable energy technologies: a novel error correction grey prediction model. IEEE Transactions on Industrial Electronics, 2019, 66(12): 9312-9325.

[14] CHEN Z, CAI Y. GM(1, 1) Residual error correction model applied to building deformation prediction. Geometrics \& Spatial Information Technology, 2010, 33(3): 198-200.

[15] XU N, DANG Y, GONG Y. Novel grey prediction model with nonlinear optimized time response method for forecasting of electricity consumption in China. Energy, 2017, 118: 473-480.

[16] MA X, LIU Z, WANG Y. Application of a novel nonlinear multivariate grey Bernoulli model to predict the tourist income of China. Journal of Computational and Applied Mathematics, 2018, 347: 84-94.

[17] DING S, XU N, YE J, et al. Estimating Chinese energy-related $\mathrm{CO}_{2}$ emissions by employing a novel discrete grey prediction model. Journal of Cleaner Production, 2020, 259: 120793.

[18] DAI J, LIU H, SUN Y, et al. An optimization method of multivariable MGM $(1, m)$ prediction model's background value. Journal of Grey System, 2018, 30(1): 221-238.

[19] WANG Y, JIE L. Improvement and application of GM(1, 1) model based on multivariable dynamic optimization. Journal of Systems Engineering and Electronics, 2020, 31(3): 593-601.

[20] XIE N, LIU S. Discrete GM(1, 1) and mechanism of grey forecasting model. Systems Engineering-Theory \& Practice, 2005, 25(1): 93-99.

[21] WANG Q, SONG X. Forecasting China's oil consumption: a comparison of novel nonlinear-dynamic grey model (GM), linear GM, nonlinear GM and metabolism GM. Energy, 2019, 183: 160-171.

[22] JIANG J, ZHANG Y, LIU C, et al. An improved nonhomogeneous discrete grey model and its application. Mathematical Problems in Engineering, 2020, 2020(3): 1-8.

[23] XIE N, ZHU C, ZHENG J. Expansion modelling of discrete grey model based on multi-factor information aggregation. Journal of Systems Engineering and Electronics, 2014, 25(5): 833-839.

[24] WU W Z, JIANG J, LI Q. A novel discrete grey model and its application. Mathematical Problems in Engineering, 2019, 2019: 9623878.

[25] SU X, HOU L, XIA L, et al. Citric acid-modulated in situ synthesis of 3D hierarchical Bi@BiOCl microsphere photocatalysts with enhanced photocatalytic performance. Journal of Materials Science, 2019, 54: 4559-4572. 